\title{
Development and statistical optimization of sustained release gastro retentive floating tablets of Cephalexin
}

\author{
Kambham VENKATESWARLU, K.B. CHANDRASEKHAR
}

\begin{abstract}
The aim of present study was to formulate the sustained release floating tablets of Cephalexin (CPL) by direct compression method and optimized by Box-behnken Response Surface Methodology (RSM). A computer aided optimization technique was employed to investigate the formulation design by Boxbehnken RSM (Design Expert Software version 8.0.7.1) to study the effect of the concentration of various polymer blends on the property of CPL gastroretentive floating tablets like floating lag time (FLT) and cumulative percent drug release (\%CDR). The independent variables investigated were polymeric concentrations (X1) and dependent variables were FLT (Y1), \%CDR (Y2) in $0.1 \mathrm{~N} \mathrm{HCl} \mathrm{(pH} \mathrm{1.2)} \mathrm{buffer.}$ Results from precompression evaluation indicated that all
\end{abstract}

the 17 and optimized formulation F18 were complied with the pharmacopoeial limits and post compression parameters were also gave satisfactory results. The predicted value for FLT and \%CDR obtained from software was compared with the experimental value of FLT and \%CDR of optimized formulation F18. It was observed that the obtained experimental results for optimized formulation were in very close agreement with the predicted values and also the optimized formulation gave good FLT and \%CDR when compared to the 17 formulations.

Keywords: Cephalexin; Design Expert Software; Floating tablets; Optimization; Response Surface Methodology.

\section{Kambham VENKATESWARLU}

Faculty of Pharmacy, Department of Pharmaceutics, JNTUA-Oil Technological and Pharmaceutical Research Institute, Jawaharlal Nehru Technological University Anantapur, Ananthapuramu-515001, Andhra Pradesh, India.

\section{K.B. CHANDRASEKHAR}

Professor of Chemistry and Director, Oil Technological and Pharmaceutical Research Institute, Jawaharlal Nehru Technological University Anantapur, Ananthapuramu-515001, Andhra Pradesh, India.

Corresponding author:

Kambham VENKATESWARLU

E-mail:k.v.reddy9441701016@gmail.com

Submitted/Gönderilme: 06.03.2016 Accepted/Kabul: 12.04.2016
Revised/Düzeltme: 08.04.2016

\section{INTRODUCTION}

Gastro retentive technology is playing a major role in revolutionizing the future of gastric retention in the pharmaceutical industry. The gastro retentive floating drug delivery system can be used as an alternative to conventional dosage forms for the class of drugs which undergoes intestinal or enzymatic degradation and generally, those drugs are acidic in nature (1). Drug absorption in the GI tract is a highly variable procedure and gastro retentive drug delivery systems prolong gastric retention of the dosage forms, extend the time for drug absorption thereby reduces drug wastage, improve bioavailability and solubility of drug which is less soluble in the high $\mathrm{pH}$ environment (2). For gastric retention, floating drug delivery system is considered to be a potential approach and considerable research has been done on CPL floating tablets (3-7).

CPL, a $\beta$-lactam antibiotic, is a broad-spectrum antibiotic for the treatment of wide variety of bacterial infections, including urinary tract infections and respiratory tract infections (3). It 
is a lipophilic weak acid with pKa values of 4.5 and is stable in gastric conditions but degrades in intestinal conditions, hence a gastro retentive dosage form would be essential to ensure better drug delivery (4). CPL has higher absorption in proximal region of the gastrointestinal tract and lesser bioavailability (35\%) mainly due to its instability in intestine and its narrow absorption window at the upper GI tract (5). This suggests that CPL is an ideal candidate for gastro retentive drug delivery system which prolongs the gastric residence time of the drug, providing prolonged drug release in upper gastro intestinal tract, where absorption of CPL is well confined.

The main objective of the present study was to formulate and optimize the sustained release gastro retentive floating tablets of CPL by direct compression method using Box-behnken response surface methodology.

\section{MATERIALS AND METHODS}

\subsection{Materials}

CPL was purchased from Orchid Chemicals and Pharmaceuticals Ltd., India. Hydroxy Propyl Methyl Cellulose (HPMC K4M) obtained from Strides Acrolabs, India, Cetyl alcohol (CA) from Loba chemie, India, Sodium Carboxy Methyl Cellulose (SCMC) from Fisher scientific, India, Microcrystalline cellulose (MCC) from Rolex chemical industries, India, Sodium lauryl sulphate (SLS) and Sodium bicarbonate (SBC) from Yarrow chemicals, India, Talc and Magnesium stearate (MS) from S.D. Fine chem., India.

\subsection{FTIR studies}

The drug-excipients compatibility was determined by $\mathrm{KBr}$ disc (pellet) method using FTIR Spectrophotometer (Bruker, USA). The discs of pure drug and its physical mixtures were prepared and scanned from 4000 to $400 \mathrm{~cm}^{-1}$. The obtained spectra of pure drug and spectra of its physical mixtures were compared to know the possible drug-excipients interactions.

\subsection{Experimental design of floating tablets of CPL}

The factors like HPMC K4M, CA and SBC were identified as critical formulation factors based on their effect or role on final dosage form from existing studies (3-7) and any variation in those factors shows a variable response on the dosage form. Hence, the careful optimization of these factors is essential to obtain a better response of the developed dosage form. In the present investigation, independent formulation variables evaluated were X1: Polymeric concentrations and dependent variables investigated were Y1: FLT in $0.1 \mathrm{~N} \mathrm{HCl}$ ( $\mathrm{pH}$ 1.2), $\mathrm{Y} 2$ : \% drug release in $0.1 \mathrm{~N} \mathrm{HCl}(\mathrm{pH} 1.2)$ buffer at the end of $8 \mathrm{~h}$. Seventeen different formulation batches of floating tablets were evaluated to determine the potential effect of those independent variables on dependent variables (8). From the existing works, the minimum and maximum effective concentrations of those variables were entered into the software; thereby it was designed 17 formulations with different polymeric concentrations (Table 1).

Table 1. Formulation of CPL gastro retentive floating tablets

\begin{tabular}{|c|c|c|c|c|c|c|c|c|c|c|c|c|c|c|c|c|c|}
\hline \multirow{2}{*}{ Ingredients } & \multicolumn{17}{|c|}{ Quantity per tablrt (mg/tablet) } \\
\hline & F1 & F2 & F3 & F4 & F5 & F6 & F7 & F8 & F9 & F10 & F11 & F12 & F13 & F14 & F15 & F16 & F17 \\
\hline CPL & 250 & 250 & 250 & 250 & 250 & 250 & 250 & 250 & 250 & 250 & 250 & 250 & 250 & 250 & 250 & 250 & 250 \\
\hline HPMC K4M & 145 & 145 & 135 & 135 & 145 & 145 & 135 & 155 & 135 & 145 & 145 & 145 & 155 & 145 & 155 & 155 & 145 \\
\hline $\mathrm{CA}$ & 40 & 50 & 50 & 40 & 40 & 40 & 40 & 30 & 30 & 30 & 40 & 50 & 40 & 40 & 40 & 50 & 30 \\
\hline SBC & 40 & 30 & 40 & 30 & 40 & 40 & 50 & 40 & 40 & 30 & 40 & 50 & 50 & 40 & 30 & 40 & 50 \\
\hline SCMC & 35 & 35 & 35 & 35 & 35 & 35 & 35 & 35 & 35 & 35 & 35 & 35 & 35 & 35 & 35 & 35 & 35 \\
\hline MCC & 23.5 & 23.5 & 23.5 & 43.5 & 23.5 & 23.5 & 23.5 & 23.5 & 43.5 & 43.5 & 23.5 & 3.5 & 3.5 & 23.5 & 23.5 & 3.5 & 13 \\
\hline SLS & 5.5 & 5.5 & 5.5 & 5.5 & 5.5 & 5.5 & 5.5 & 5.5 & 5.5 & 5.5 & 5.5 & 5.5 & 5.5 & 5.5 & 5.5 & 5.5 & 5.5 \\
\hline Talc & 5.5 & 5.5 & 5.5 & 5.5 & 5.5 & 5.5 & 5.5 & 5.5 & 5.5 & 5.5 & 5.5 & 5.5 & 5.5 & 5.5 & 5.5 & 5.5 & 5.5 \\
\hline MS & 5.5 & 5.5 & 5.5 & 5.5 & 5.5 & 5.5 & 5.5 & 5.5 & 5.5 & 5.5 & 5.5 & 5.5 & 5.5 & 5.5 & 5.5 & 5.5 & 5.5 \\
\hline Total weight & 550 & 550 & 550 & 550 & 550 & 550 & 550 & 550 & 550 & 550 & 550 & 550 & 550 & 550 & 550 & 550 & 550 \\
\hline
\end{tabular}




\subsection{Preparation of CPL floating tablets}

Tablets containing $250 \mathrm{mg}$ of CPL were prepared according to the design depicted in table 1 by direct compression method. The excipients were chosen after the comprehensive drug excipient interaction studies and those were namely release retarding polymer (s) like HPMC K4M, CA and SCMC, SBC (gas generating agent), SLS and MCC (filler) were passed through sieve no.60, separately. Mixing of the ingredients was carried out by using a mortar and pestle for $10 \mathrm{~min}$. Lubricant (Magnesium stearate) and glidant (Talc) previously passed through sieve no. 60 were added to the mixture and mixing was continued for additional $5 \mathrm{~min}$. Finally, $550 \mathrm{mg}$ of each mixture was weighed and fed manually in to the die of a 10 station tablet punching machine using $12 \mathrm{~mm}$ round shaped punches to produce the required tablets (RIMEK Rotary Tablet Punching machine, India). The hardness was adjusted to $7 \mathrm{~kg} / \mathrm{cm}^{2}$.

\subsection{Evaluation studies}

\subsubsection{Precompression evaluation studies}

The powder blends were evaluated for the precompression parameters like bulk density, tapped density, compressibility index (Carr's Index), Hausner's ratio and angle of repose (9$10)$.

\subsubsection{Post compression evaluation studies}

The tablets were evaluated for finished product quality control tests like thickness, weight variation, hardness, friability and drug content uniformity (11-12).

\subsubsection{Tablet floating behavior}

The floating behavior of the tablets was visually determined in triplicate, according to the FLT method. Briefly, a tablet was placed in a glass beaker containing $100 \mathrm{ml}$ of $0.1 \mathrm{~N} \mathrm{HCl}$ $(\mathrm{pH} 1.2)$ and maintained in a water bath at $37 \pm 0.5^{\circ} \mathrm{C}$, thereby the floating time was measured (13-14).

\subsubsection{Swelling studies}

The extent of swelling is measured in terms of \% of weight gained by the tablet. One tablet from each formulation was weighed $\left(\mathrm{w}_{1}\right)$ and kept in glass beaker containing $100 \mathrm{ml}$ of
$0.1 \mathrm{~N} \mathrm{HCl}(\mathrm{pH} 1.2)$ buffer. At the end of the specified time intervals, tablets were withdrawn from the glass beaker and therefore the excess buffer was blotted with the tissue paper by taking care to avoid the surface erosion from tablet and swollen tablet was then reweighed $\left(\mathrm{w}_{2}\right)(15)$. The $\%$ of weight gained by the tablet was calculated by using following formula as.

\subsubsection{In-vitro dissolution studies}

In-vitro dissolution study of CPL was performed using USP dissolution apparatus, type II (Paddle method) (Lab India 8 basket dissolution apparatus, India) at $37^{\circ} \mathrm{C} \pm 0.5^{\circ} \mathrm{C}$ and the paddle was set to rotate at a speed of $50 \mathrm{rpm}$. The tablets were placed in the dissolution apparatus containing $900 \mathrm{ml}$ of $0.1 \mathrm{~N} \mathrm{HCl}(\mathrm{pH} 1.2)$ buffer as dissolution medium. Samples were withdrawn $(10 \mathrm{ml})$ and replaced with an equal amount of fresh dissolution medium at particular time intervals, samples were immediately filtered through Whatmann filter paper and diluted with the dissolution media. The absorbances of these diluted samples were noted at $\lambda \max 256$ $\mathrm{nm}$ using UV-Visible Spectrophotometer (Lab India 1700 UV-Visible spectrophotometer, India) (16-17).

\subsection{Kinetics studies}

The release kinetics of the drug was described by fitting the obtained in-vitro dissolution data into various kinetic models like zero order, first order, Higuchi's and Korsmeyer-Peppas models (18-19).

\section{RESULTS AND DISCUSSION}

\subsection{FTIR Studies}

FTIR spectrum of pure drug and its physical mixtures were studied. The major IR peaks observed for pure CPL at 1758.22, 3272.98, 1689.58, 1454.85 and $1594.85 \mathrm{~cm}^{-1}$ were mainly because of $\mathrm{C}=\mathrm{O}$ stretching, $\mathrm{N}-\mathrm{H}$ stretching, $\mathrm{C}=\mathrm{O}$ stretching, $\mathrm{C}=\mathrm{C}$ stretching and $\mathrm{N}-\mathrm{H}$ bending respectively. In the present study, it was observed that there were no major shifts in its individual characteristic peaks; hence, it indicates that there were no incompatibility issues between drug and polymers used (Figure 1). 

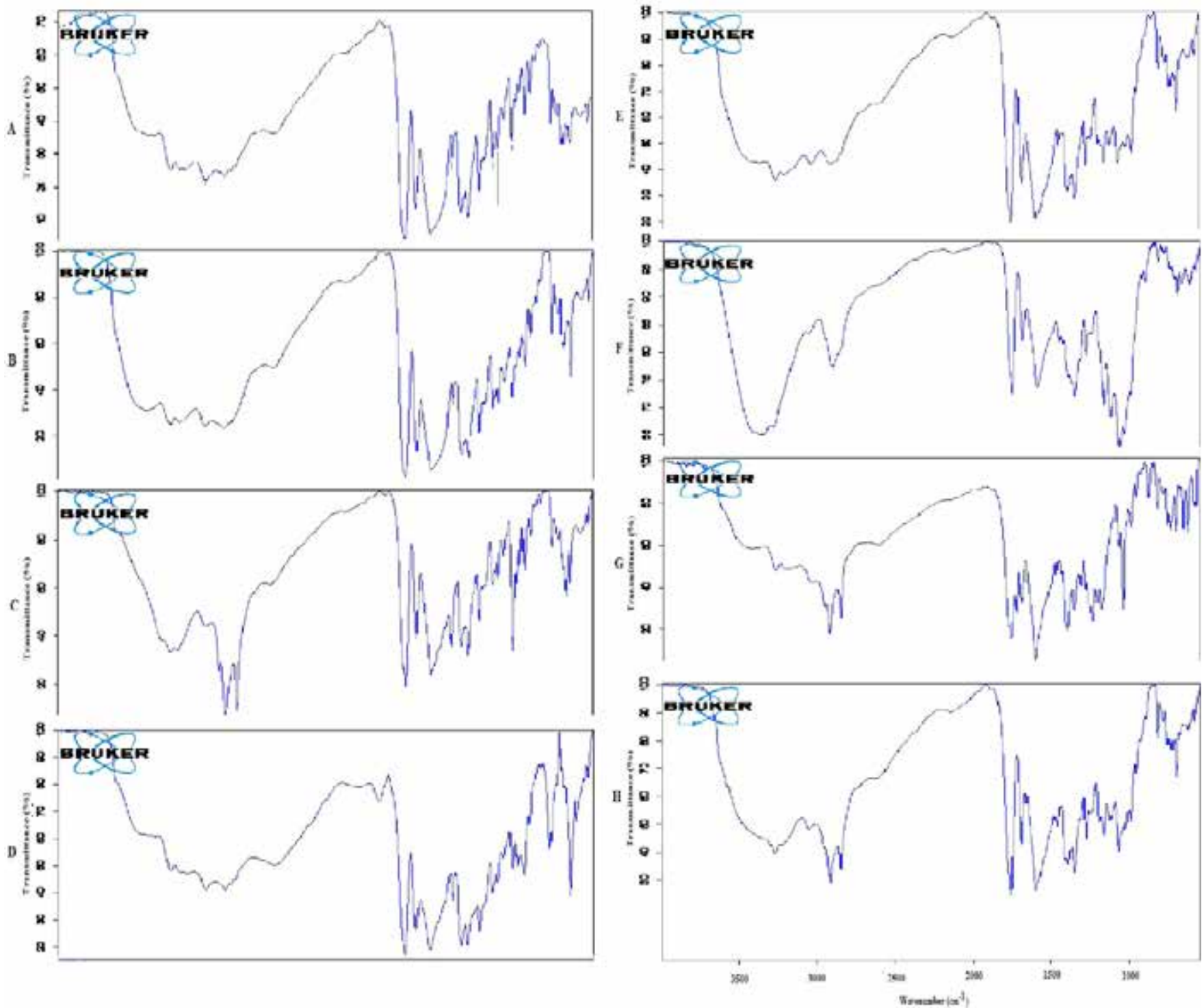

Figure 1. FTIR spectra CPL with excipients. A) FTIR spectra of pure CPL, B) FTIR spectra of CPL + HPMC K4M, C) FTIR spectra of CPL + Cetyl alcohol, D) FTIR spectra of CPL + SBC, E) FTIR spectra of CPL + SCMC, F) FTIR spectra of CPL + MCC, G) FTIR spectra of CPL +SLS, H) FTIR spectra of CPL optimized formulation (F-18)

\subsection{Precompression evaluation}

The powder blends of formulations F1-F17 have the bulk density ranged from $0.492 \pm 0.0087$ to $0.512 \pm 0.0056$ and tapped density from $0.587 \pm 0.012$ to $0.618 \pm 0.0080$. The compressibility index values less than 10, 11-15, 16-20, 21$25,26-31,32-37$ and greater than 38 indicates excellent, good, fair, possible, poor, very poor and very very poor flow respectively and powder blends of all the formulations [F1-F17] developed in the formulation development phase were found to be possessing the good to fair flowability i.e.
13.549 to $19.626 \%$. The ideal Hausner's ratio values of 1.0 $1.11,1.12-1.18,1.19-1.25,1.26-1.34,1.35-1.45,1.46-1.59$ and greater 1.60 indicates excellent, good, fair, possible, poor, very poor and very very poor flow respectively and powder blends of all the formulations were found to be possessing the fair to passable flowability i.e. 1.156 to 1.244 . The ideal angle of repose values of less than 25, 25-30, 30-40 and greater than 40 indicates excellent, good, passable and very poor respectively and the experimental values of all the formulations were ranged from $26.581^{\circ}$ to $30.504^{\circ}$ indicates good to passable flowability (Table 2). 
Table 2. Evaluation of precompression parameters

\begin{tabular}{|c|c|c|c|c|c|}
\hline Formulation & $\begin{array}{l}\text { Bulk density } \\
\text { (gm/cc) }\end{array}$ & $\begin{array}{c}\text { Tapped density } \\
(\mathrm{gm} / \mathrm{cc})\end{array}$ & $\begin{array}{l}\text { Carr's index } \\
\text { (I) }\end{array}$ & Hausner's ratio & $\begin{array}{c}\text { Angle of repose } \\
\left({ }^{0}\right)\end{array}$ \\
\hline $\begin{array}{c}\text { CPL } \\
\text { (pure drug) }\end{array}$ & $0.59 \pm 0.012$ & $0.76 \pm 0.021$ & $21.72 \pm 1.99$ & $1.27 \pm 0.032$ & $36.17 \pm 0.64$ \\
\hline $\mathrm{F} 1$ & $0.53 \pm 0.006$ & $0.61 \pm 0.008$ & $13.54 \pm 0.33$ & $1.15 \pm 0.002$ & $30.50 \pm 1.03$ \\
\hline $\mathrm{F} 2$ & $0.50 \pm 0.004$ & $0.62 \pm 0.014$ & $19.202 .34 \pm$ & $1.23 \pm 0.036$ & $28.82 \pm 2.84$ \\
\hline F3 & $0.49 \pm 0.008$ & $0.61 \pm 0.013$ & $19.63 \pm 1.64$ & $1.24 \pm 0.025$ & $26.58 \pm 0.54$ \\
\hline F4 & $0.49 \pm 0.009$ & $0.60 \pm 0.013$ & $18.171 .68 \pm$ & $1.22 \pm 0.025$ & $28.17 \pm 0.98$ \\
\hline F5 & $0.53 \pm 0.006$ & $0.61 \pm 0.008$ & $13.54 \pm 0.15$ & $1.15 \pm 0.002$ & $30.501 .03 \pm$ \\
\hline F6 & $0.53 \pm 0.006$ & $0.61 \pm 0.008$ & $13.54 \pm 0.15$ & $1.15 \pm 0.002$ & $30.501 .03 \pm$ \\
\hline F7 & $0.49 \pm 0.009$ & $0.60 \pm 0.007$ & $16.96 \pm 0.78$ & $1.20 \pm 0.011$ & $27.211 .08 \pm$ \\
\hline F8 & $0.49 \pm 0.046$ & $0.59 \pm 0.013$ & $17.813 .25 \pm$ & $1.21 \pm 0.048$ & $27.701 .89 \pm$ \\
\hline F9 & $0.51 \pm 0.005$ & $0.61 \pm 0.013$ & $16.442 .09 \pm$ & $1.19 \pm 0.030$ & $30.411 .60 \pm$ \\
\hline F10 & $0.51 \pm 0.005$ & $0.60 \pm 0.016$ & $15.80 \pm 2.77$ & $1.18 \pm 0.039$ & $25.90 \pm 1.07$ \\
\hline F11 & $0.53 \pm 0.006$ & $0.61 \pm 0.008$ & $13.540 .15 \pm$ & $1.15 \pm 0.002$ & $30.501 .03 \pm$ \\
\hline F12 & $0.51 \pm 0.005$ & $0.61 \pm 0.008$ & $14.64 \pm 1.03$ & $1.17 \pm 0.014$ & $28.03 \pm 0.96$ \\
\hline F13 & $0.50 \pm 0.005$ & $0.58 \pm 0.012$ & $14.26 \pm 2.71$ & $1.16 \pm 0.036$ & $28.50 \pm 1.167$ \\
\hline F14 & $0.53 \pm 0.006$ & $0.61 \pm 0.008$ & $13.54 \pm 0.15$ & $1.15 \pm 0.002$ & $30.50 \pm 1.03$ \\
\hline F15 & $0.50 \pm 0.009$ & $0.61 \pm 0.013$ & $18.84 \pm 2.88$ & $1.22 \pm 0.044$ & $28.812 .08 \pm$ \\
\hline F16 & $0.50 \pm 0.005$ & $0.58 \pm 0.019$ & $14.973 .60 \pm$ & $1.17 \pm 0.049$ & $29.061 .05 \pm$ \\
\hline F17 & $0.50 \pm 0.005$ & $0.59 \pm 0.013$ & $15.60 \pm 2.69$ & $1.18 \pm 0.038$ & $26.89 \pm 1.05$ \\
\hline
\end{tabular}

All the results were expressed in mean $\pm S D(n=3)$

\subsection{Post compression evaluation}

Weight variation test revealed that all the formulations [F1F17] developed in the formulation development phase were complied with the official limits (if the tablet weight is greater than $324 \mathrm{mg}$, the maximum difference allowed is $\pm 10 \mathrm{mg}$ ) and ranged from $550.333 \pm 0.288 \mathrm{mg}$ to $551 \pm 0.5 \mathrm{mg}$. Hardness of all the formulations was ranged from $6.65 \pm 0.060 \mathrm{~kg} / \mathrm{cm}^{2}$ to $6.943 \pm 0.260 \mathrm{~kg} / \mathrm{cm}^{2}$ and thickness ranged from $4.46 \pm 0.026$ to $4.496 \pm 0.050 \mathrm{~mm}$. The ideal friability value is less than $1 \%$ and the obtained experimental values were ranged from $0.427 \pm 0.082 \%$ to $0.894 \pm 0.016 \%$, hence the friability test was satisfied. The maximum difference allowed for drug content is \pm 5 and it was ranged from $97.254 \pm 1.113 \%$ to $99.999 \pm 4.043 \%$ (Table 3).

\subsection{Floating behavior}

On immersion in $0.1 \mathrm{~N} \mathrm{HCl}(\mathrm{pH} 1.2)$ at $37^{\circ} \mathrm{C}$, it expands and $\mathrm{CO}_{2}$ was formed within the tablets thereby floated and remained buoyant without disintegration. Formulations consists of high concentration of HPMC K4M (F-8) showed good FLT and TFT, which might be due to the rapid hydration of the polymer, thereby it forms gelatinous layer when exposed to aqueous medium. This gelatinous layer prevents the escape of $\mathrm{CO}_{2}$ from dosage form thereby decreases the density which leads to floating of tablets within a short period of time (Table 3) (19). 
Table 3. Evaluation of post compression parameters

\begin{tabular}{|c|c|c|c|c|c|c|c|}
\hline Formulation & $\begin{array}{c}\text { Weight variation } \\
(\mathbf{m g})\end{array}$ & $\begin{array}{l}\text { Hardness }(\mathbf{k g} / \\
\left.\mathbf{c m}^{2}\right)\end{array}$ & Thickness (mm) & $\begin{array}{c}\text { Friability } \\
(\%)\end{array}$ & $\%$ Drug content & TFT (h) & $\begin{array}{l}\text { FLT } \\
\text { (sec) }\end{array}$ \\
\hline $\mathrm{F} 1$ & $550.5 \pm 0.50$ & $6.73 \pm 0.21$ & $4.46 \pm 0.032$ & $0.50 \pm 0.04$ & $98.33 \pm 1.48$ & $>12$ & 15.81 \\
\hline $\mathrm{F} 2$ & $550.3 \pm 0.28$ & $6.84 \pm 0.17$ & $4.46 \pm 0.026$ & $0.89 \pm 0.01$ & $99.11 \pm 0.72$ & $>12$ & 14.69 \\
\hline F3 & $550.6 \pm 0.28$ & $6.83 \pm 0.18$ & $4.49 \pm 0.050$ & $0.65 \pm 0.10$ & $98.62 \pm 1.79$ & $>12$ & 8.17 \\
\hline $\mathrm{F} 4$ & $550.8 \pm 1.04$ & $6.94 \pm 0.26$ & $4.47 \pm 0.020$ & $0.54 \pm 0.07$ & $98.82 \pm 1.06$ & $>12$ & 9.72 \\
\hline F5 & $550.5 \pm 0.50$ & $6.73 \pm 0.21$ & $4.46 \pm 0.032$ & $0.50 \pm 0.04$ & $98.33 \pm 1.48$ & $>12$ & 15.81 \\
\hline F6 & $550.5 \pm 0.50$ & $6.73 \pm 0.21$ & $4.46 \pm 0.032$ & $0.50 \pm 0.04$ & $98.33 \pm 1.48$ & $>12$ & 15.81 \\
\hline F7 & $550.6 \pm 0.28$ & $6.66 \pm 0.08$ & $4.46 \pm 0.032$ & $0.53 \pm 0.13$ & $97.54 \pm 2.73$ & $>12$ & 9.44 \\
\hline F8 & $551.0 \pm 0.50$ & $6.73 \pm 0.12$ & $4.46 \pm 0.013$ & $0.53 \pm 0.07$ & $97.25 \pm 1.11$ & $>12$ & 19.63 \\
\hline F9 & $550.3 \pm 0.57$ & $6.70 \pm 0.17$ & $4.45 \pm 0.020$ & $0.63 \pm 0.04$ & $98.72 \pm 2.44$ & $>12$ & 12.52 \\
\hline F10 & $550.3 \pm 0.28$ & $6.73 \pm 0.11$ & $4.46 \pm 0.025$ & $0.57 \pm 0.11$ & $99.99 \pm 4.04$ & $>12$ & 16.22 \\
\hline F11 & $550.5 \pm 0.50$ & $6.73 \pm 0.21$ & $4.46 \pm 0.032$ & $0.50 \pm 0.04$ & $98.33 \pm 1.48$ & $>12$ & 15.81 \\
\hline $\mathrm{F} 12$ & $550.3 \pm 0.28$ & $6.85 \pm 0.09$ & $4.46 \pm 0.015$ & $0.49 \pm 0.05$ & $99.41 \pm 2.05$ & $>12$ & 9.89 \\
\hline F13 & $550.5 \pm 0.52$ & $6.69 \pm 0.14$ & $4.47 \pm 0.020$ & $0.44 \pm 0.07$ & $99.503 .53 \pm$ & $>12$ & 20.25 \\
\hline F14 & $550.5 \pm 0.50$ & $6.73 \pm 0.21$ & $4.46 \pm 0.032$ & $0.50 \pm 0.04$ & $98.33 \pm 1.48$ & $>12$ & 15.81 \\
\hline $\mathrm{F} 15$ & $550.6 \pm 0.28$ & $6.76 \pm 0.13$ & $4.49 \pm 0.050$ & $0.42 \pm 0.08$ & $98.72 \pm 2.94$ & $>12$ & 22.88 \\
\hline F16 & $550.60 .57 \pm$ & $6.65 \pm 0.06$ & $4.48 \pm 0.030$ & $0.58 \pm 0.05$ & $98.03 \pm 0.89$ & $>12$ & 19.94 \\
\hline F17 & $550.8 \pm 0.57$ & $6.66 \pm 0.16$ & $4.47 \pm 0.020$ & $0.63 \pm 0.07$ & $99.60 \pm 0.61$ & $>12$ & 14.54 \\
\hline
\end{tabular}

All the results were expressed in mean $\pm \operatorname{SD}(n=3)$

\subsection{Swelling study}

The swelling index was calculated with respect to the time and results were represented in table 4 . As the time increases, the swelling index was also increased, because weight gain by tablet was increased proportionally with rate of hydration. Later on, it decreased gradually due to dissolution of outermost gelled layer of the tablet into dissolution medium (20). Water uptake studies showed that the formulation with higher percentage of HPMC K4M imbibed more water and was swollen to greater extent than formulation with low percentage of HPMC K4M. Swelling index of all the formulations increased upto $6^{\text {th }} \mathrm{h}$ and subsequently it was decreased at $8^{\text {th }} \mathrm{h}$. The swelling index of the formulation F8 was found to be maximum throughout the study (Table 14). The swelling index of all the formulations is increases with increase in the concentration of HPMC K4M and decreases with increase in the concentration of CA due to its hydrophobic nature (21). Incorporation of varying amounts of SBC in the formulation had shown no significant impact on the swelling index. Formulations (F3, F4, F7 and F9) with low concentration of HPMC K4M swelled instantly which did not persist due to subsequent erosion which was supported by the kinetic studies where the $\mathrm{r}^{2}$ value was better to the Higuchi model except F9. 
Table 4. Mean swelling indices of all formulations

\begin{tabular}{|c|c|c|c|c|c|c|c|c|}
\hline Formulation & $\begin{array}{c}\text { At } \\
1^{\text {st }} \mathbf{h}\end{array}$ & $\begin{array}{c}\text { At } \\
2^{\text {nd }} h\end{array}$ & $\begin{array}{c}\text { At } \\
3^{\text {rd }} \mathbf{h}\end{array}$ & $\begin{array}{c}\text { At } \\
4^{\text {th }} h\end{array}$ & $\begin{array}{c}\text { At } \\
5^{\text {th }} \mathbf{h}\end{array}$ & $\begin{array}{c}\text { At } \\
6^{\text {th }} h\end{array}$ & $\begin{array}{c}\text { At } \\
7^{\text {th }} \mathbf{h}\end{array}$ & $\begin{array}{c}\text { At } \\
8^{\text {th }} \mathbf{h}\end{array}$ \\
\hline $\mathrm{F} 1$ & $55.57 \pm 0.93$ & $87.75 \pm 0.45$ & $107.57 \pm 0.27$ & $110.90 \pm 0.18$ & $114.12 \pm 0.45$ & $125.51 \pm 0.27$ & $131.00 \pm 0.11$ & $121.63 \pm 0.18$ \\
\hline $\mathrm{F} 2$ & $58.24 \pm 0.27$ & $83.69 \pm 0.27$ & $100.24 \pm 0.58$ & $111.81 \pm 0.36$ & $118.30 \pm 0.55$ & $120 \pm 0.36$ & $112.77 \pm 0.27$ & $107.33 \pm 0.45$ \\
\hline F3 & $54.60 \pm 0.27$ & $73.21 \pm 0.45$ & $85.51 \pm 0.45$ & $88.90 \pm 0.65$ & $120.12 \pm 0.37$ & $121.21 \pm 0.13$ & $116.33 \pm 0.33$ & $109.15 \pm 0.45$ \\
\hline $\mathrm{F} 4$ & $60 \pm 0.36$ & $71.03 \pm 0.37$ & $96.36 \pm 0.36$ & $98.18 \pm 0.18$ & $107.33 \pm 0.27$ & $114.66 \pm 0.37$ & $107.44 \pm 0.41$ & $100.18 \pm 0.48$ \\
\hline F5 & $55.57 \pm 0.93$ & $87.75 \pm 0.45$ & $107.57 \pm 0.27$ & $110.90 \pm 0.18$ & $114.12 \pm 0.45$ & $125.51 \pm 0.27$ & $131.00 \pm 0.11$ & $121.63 \pm 0.18$ \\
\hline F6 & $55.57 \pm 0.93$ & $87.75 \pm 0.45$ & $107.57 \pm 0.27$ & $110.90 \pm 0.18$ & $114.12 \pm 0.45$ & $125.51 \pm 0.27$ & $131.09 \pm 0.181$ & $121.63 \pm 0.18$ \\
\hline F7 & $54.54 \pm 0.36$ & $72.66 \pm 0.45$ & $87.33 \pm 0.27$ & $94.66 \pm 0.55$ & $126.24 \pm 0.73$ & $130.96 \pm 0.27$ & $123.69 \pm 0.45$ & $114.36 \pm 0.65$ \\
\hline F8 & $62.12 \pm 0.27$ & $80.06 \pm 0.63$ & $103.51 \pm 0.37$ & $110.96 \pm 0.27$ & $131.09 \pm 0.65$ & $132.84 \pm 0.55$ & $123.81 \pm 0.48$ & $116.30 \pm 0.45$ \\
\hline F9 & $53.15 \pm 0.45$ & $74.60 \pm 0.45$ & $94.54 \pm 0.36$ & $104.06 \pm 0.45$ & $118.18 \pm 0.36$ & $120.66 \pm 0.89$ & $118.60 \pm 0.37$ & $112.78 \pm 0.45$ \\
\hline F10 & $56.78 \pm 0.37$ & $84.06 \pm 0.45$ & $102.72 \pm 0.12$ & $112.78 \pm 0.27$ & $114.66 \pm 0.37$ & $125.44 \pm 0.11$ & $119.93 \pm 0.45$ & $108.54 \pm 0.10$ \\
\hline $\mathrm{F} 11$ & $55.57 \pm 0.93$ & $87.75 \pm 0.45$ & $107.57 \pm 0.27$ & $110.90 \pm 0.18$ & $114.12 \pm 0.45$ & $125.55 \pm 0.27$ & $131.09 \pm 0.18$ & $121.63 \pm 0.18$ \\
\hline $\mathrm{F} 12$ & $60.18 \pm 0.18$ & $87.45 \pm 0.18$ & $102.24 \pm 0.45$ & $105.39 \pm 0.45$ & $120.06 \pm 0.27$ & $125.55 \pm 0.38$ & $123.69 \pm 0.45$ & $116.12 \pm 0.27$ \\
\hline F13 & $56.60 \pm 0.27$ & $89.15 \pm 0.27$ & $105.87 \pm 0.45$ & $107.21 \pm 0.45$ & $129.09 \pm 0.36$ & $138.66 \pm 0.47$ & $136.30 \pm 0.45$ & $131.15 \pm 0.27$ \\
\hline F14 & $55.57 \pm 0.93$ & $87.75 \pm 0.45$ & $107.57 \pm 0.27$ & $110.90 \pm 0.18$ & $114.12 \pm 0.45$ & $125.55 \pm 0.27$ & $131.09 \pm 0.18$ & $121.63 \pm 0.18$ \\
\hline F15 & $57.45 \pm 0.18$ & $92.36 \pm 0.48$ & $102.18 \pm 0.36$ & $105.51 \pm 0.45$ & $122.30 \pm 0.45$ & $123.77 \pm 0.15$ & $129.09 \pm 0.58$ & $120.06 \pm 0.27$ \\
\hline F16 & $51.09 \pm 0.18$ & $74.60 \pm 0.27$ & $104 \pm 0.48$ & $105.57 \pm 0.37$ & $107.21 \pm 0.27$ & $118.66 \pm 0.47$ & $124.06 \pm 0.37$ & $118.72 \pm 0.54$ \\
\hline F17 & $54.54 \pm 0.18$ & $85.51 \pm 0.45$ & $103.75 \pm 0.37$ & $107.27 \pm 0.54$ & $111.03 \pm 0.55$ & $114.88 \pm 0.68$ & $151.09 \pm 0.48$ & $145.69 \pm 0.27$ \\
\hline
\end{tabular}

All the results were expressed in mean $\pm \mathrm{SD}(\mathrm{n}=3)$

\subsection{In-vitro drug release studies}

From the release profiles, it was concluded that the variation in concentrations of polymer from $\mathrm{F} 1$ to $\mathrm{F} 17$ had variable effect on drug release. The effect of HPMC K4M and CA could be observed at constant SCMC level. HPMC K4M with higher molecular weight forms gel of higher viscosity (4000cps) compared to SCMC (not less than 2000cps). Formulations containing lower limit of HPMC K4M showed early release in dissolution medium but as increase in the concentration of CA causes decrease in the drug release due to its water repelling capacity and the formulation F9 showed maximum drug release of $96.35 \%$ (Table 5). 


\begin{tabular}{|c|c|c|c|c|c|c|c|}
\hline$\hat{\vec{I}}$ & 0 & 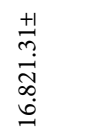 & 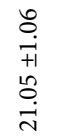 & 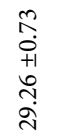 & $\begin{array}{l}\infty \\
\stackrel{\infty}{+} \\
++1 \\
\infty \\
\infty \\
\dot{f} \\
\dot{f}\end{array}$ & $\begin{array}{l}\stackrel{+}{2} \\
\stackrel{2}{\vec{D}} \\
\stackrel{0}{\circ} \\
\stackrel{+}{+}\end{array}$ & $\begin{array}{l}\mathscr{n} \\
\alpha \\
+ \\
+1 \\
+ \\
+ \\
+ \\
\infty\end{array}$ \\
\hline$\stackrel{0}{\underline{I}}$ & 0 & 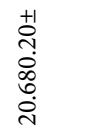 & 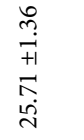 & 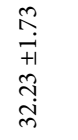 & 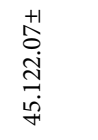 & $\begin{array}{l}+1 \\
\stackrel{+1}{10} \\
\stackrel{7}{7} \\
\dot{8}\end{array}$ & $\begin{array}{l}+1 \\
\stackrel{+}{0} \\
\dot{\vec{I}} \\
\infty \\
\dot{i}\end{array}$ \\
\hline$\frac{n}{a}$ & 0 & $\begin{array}{l}+1 \\
\stackrel{+}{0} \\
0 \\
\Delta \\
\text { in }\end{array}$ & $\begin{array}{l}0 \\
\mathscr{n} \\
0 \\
+1 \\
0 \\
\infty \\
\stackrel{+}{N}\end{array}$ & \begin{tabular}{l}
$\hat{N}$ \\
$\stackrel{+}{+1}$ \\
\multirow{y}{*}{} \\
$\stackrel{d}{+}$
\end{tabular} & 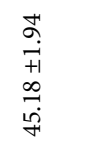 & 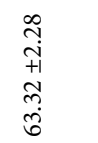 & $\begin{array}{l}\infty \\
\infty \\
0 \\
+1 \\
0 \\
0 \\
0 \\
\infty\end{array}$ \\
\hline$\underset{I}{I}$ & 0 & 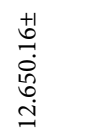 & 占 & $\begin{array}{l}\stackrel{\overbrace{}}{ت} \\
\stackrel{+}{+}\end{array}$ & $\begin{array}{l}+1 \\
\stackrel{+}{0} \\
\stackrel{2}{+} \\
\stackrel{0}{0} \\
\stackrel{0}{0} \\
i n\end{array}$ & $\begin{array}{l}+1 \\
0 \\
0 \\
0 \\
0 \\
0 \\
0 \\
0\end{array}$ & $\begin{array}{l}\underset{+}{+} \\
\stackrel{D}{D} \\
\infty \\
\infty \\
\infty \\
\infty\end{array}$ \\
\hline$\stackrel{m}{ \pm}$ & 0 & 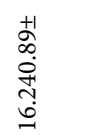 & 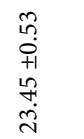 & 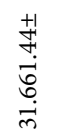 & 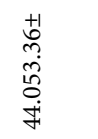 & 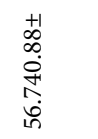 & $\underset{\substack{+\stackrel{+}{N}}}{\stackrel{+}{*}}$ \\
\hline$\underset{I}{I}$ & 0 & 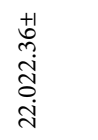 & $\begin{array}{ll}\overrightarrow{+} & \stackrel{\sigma}{\sim} \\
\overrightarrow{+}\end{array}$ & 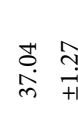 & 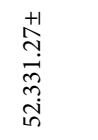 & $\begin{array}{l}+1 \\
\stackrel{+}{a} \\
\dot{a} \\
\dot{0} \\
\dot{0} \\
\dot{0}\end{array}$ & 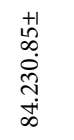 \\
\hline 玨 & 0 & 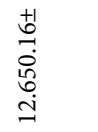 & $\begin{array}{l}\stackrel{2}{\alpha} \underset{+}{+} \\
\stackrel{+}{+}\end{array}$ & 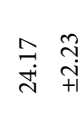 & $\begin{array}{l}+1 \\
\infty \\
\stackrel{\infty}{+} \\
\stackrel{0}{0} \\
\stackrel{0}{0} \\
i n\end{array}$ & $\begin{array}{l}+1 \\
0 \\
0 \\
0 \\
0 \\
0 \\
0\end{array}$ & $\begin{array}{l}+ \\
\stackrel{+}{0} \\
\stackrel{.}{0} \\
\infty \\
\infty \\
\infty \\
\infty\end{array}$ \\
\hline 옴 & 0 & $\begin{array}{l}+1 \\
\stackrel{0}{0} \\
\stackrel{0}{0} \\
\stackrel{0}{=}\end{array}$ & 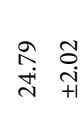 & $\begin{array}{ll}\hat{b} & \infty \\
\dot{m} & +\end{array}$ & 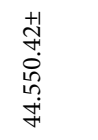 & 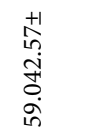 & 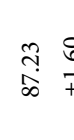 \\
\hline के & 0 & 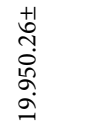 & 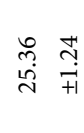 & 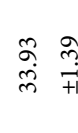 & 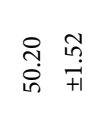 & 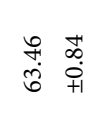 & 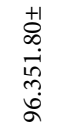 \\
\hline 䎹 & 0 & 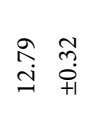 & 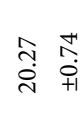 & $\stackrel{\infty}{n}_{m}^{\infty}$ & 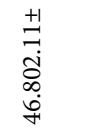 & 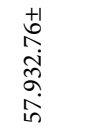 & $\begin{array}{c}\infty \\
\underset{1}{1} \\
\infty \\
\infty\end{array}$ \\
\hline 圭 & 0 & $\begin{array}{ll}\vec{J} & \vec{m} \\
\dot{I} & \stackrel{+}{+}\end{array}$ & $\begin{array}{l}\hat{\circ} \overrightarrow{0} \\
\stackrel{+}{+}\end{array}$ & 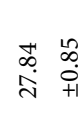 & 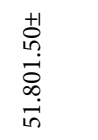 & 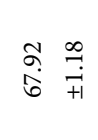 & 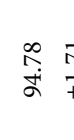 \\
\hline L & 0 & 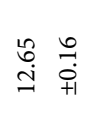 & 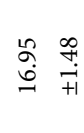 & 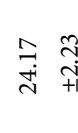 & 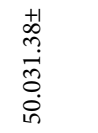 & $\begin{array}{l}+1 \\
0 \\
0 \\
0 \\
0 \\
0 \\
0\end{array}$ & $\begin{array}{c}\infty \\
\infty \\
\infty \\
\infty\end{array}$ \\
\hline 号 & 0 & 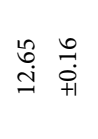 & 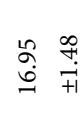 & $\underset{\vec{i}}{\stackrel{\tilde{I}}{+}}$ & $\begin{array}{l}++1 \\
\infty \\
\stackrel{2}{+} \\
\stackrel{0}{0} \\
\stackrel{0}{0}\end{array}$ & $\begin{array}{l}+1 \\
\text { ते } \\
i \\
0 \\
0 \\
0 \\
0\end{array}$ & $\begin{array}{l}\infty \\
\infty \\
\infty \\
\infty\end{array}$ \\
\hline $\mathbb{I}$ & 0 & 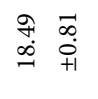 & 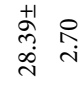 & 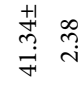 & $\begin{array}{ll}+1 & \infty \\
0 \\
0 \\
0 \\
\text { in }\end{array}$ & 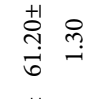 & $\begin{array}{l}+1 \\
\hat{n} \\
\alpha \\
\alpha\end{array}$ \\
\hline$M$ & 0 & 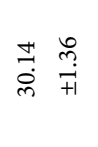 & 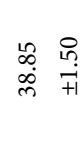 & $\frac{m}{9} \frac{n}{7}$ & 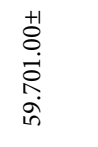 & 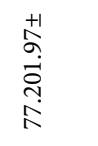 & $\begin{array}{c}+ \\
\dot{0} \\
\infty \\
\infty\end{array}$ \\
\hline $\mathbb{I}$ & 0 & $\begin{array}{ll}\vec{y} & \text { Pे } \\
\stackrel{+}{+}\end{array}$ & 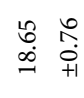 & $\underset{\sim}{\stackrel{m}{\sim}} \stackrel{+}{+}$ & $\begin{array}{ll}3 \\
\stackrel{f}{f} \\
\stackrel{+}{+} \underset{+1}{+}\end{array}$ & 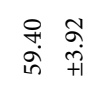 & 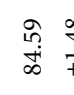 \\
\hline 左 & 0 & $\begin{array}{ll}\stackrel{0}{0} & 0 \\
\stackrel{0}{+} & + \\
+\end{array}$ & 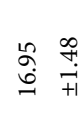 & $\underset{\vec{i}}{\stackrel{\pi}{ \pm}}$ & 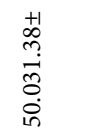 & 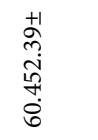 & $\begin{array}{l}\infty \\
\infty \\
\infty \\
\infty\end{array}$ \\
\hline$\stackrel{\Xi}{\Xi} \Xi$ & $\circ$ & $\stackrel{n}{0}$ & - & $\sim$ & $r$ & 0 & $\infty$ \\
\hline
\end{tabular}




\subsection{In-vitro drug release kinetics}

The mechanism of drug release for the dissolution data was determined by finding the $\mathrm{r}^{2}$ value for each kinetic model viz. zero order, first order, Higuchi's, and KorsmeyerPeppas models. For tablets, an ' $n$ ' value near to 0.45 indicates diffusion controlled drug release and an ' $n$ ' value 0.89 or near to 1 indicates swelling-controlled drug release. The intermediate values of $\mathrm{n}$ between 0.45 and 1 can be regarded as an indicator for both the phenomena (anomalous transport). For the formulations F1, F2, F4,
F5-F11, F14 and F17, the $\mathrm{r}^{2}$ value of zero order is very near to one than other kinetic models. Thus, it can be said that the drug release follows zero-order kinetics. The $\mathrm{r}^{2}$ value of formulations F3, F12, F13, F15 and F16 was found to very near to one and they follow First order kinetics. The ' $n$ ' values of Korsmeyer-Peppas model of the formulations (F1, F2 and F4-F17) have the ' $n$ ' value in the range of 0.450.89 , thus they follow the Non-Fickian transport and the ' $n$ ' value of formulation F-3 is below 0.45 which indicates the Fickian transport (Table 6).

Table 6. In-vitro drug release kinetics of all formulations

\begin{tabular}{|c|c|c|c|c|c|}
\hline Formulation & $\begin{array}{c}\text { Zero order } \\
\mathbf{r}^{2}\end{array}$ & $\begin{array}{c}\text { First order } \\
\mathbf{r}^{2}\end{array}$ & $\begin{array}{c}\text { Higuchi } \\
\mathbf{r}^{2}\end{array}$ & $\begin{array}{c}\text { Korsmeyer-Peppas } \\
\text { Diffusion exponent (n) }\end{array}$ & Drug release mechanism \\
\hline $\mathrm{F} 1$ & 0.968 & 0.940 & 0.929 & 0.777 & Non-Fickian transport \\
\hline $\mathrm{F} 2$ & 0.967 & 0.899 & 0.906 & 0.729 & Non-Fickian transport \\
\hline F3 & 0.625 & 0.949 & 0.974 & 0.393 & Fickian transport \\
\hline $\mathrm{F} 4$ & 0.847 & 0.816 & 0.949 & 0.507 & Non-Fickian transport \\
\hline F5 & 0.968 & 0.940 & 0.929 & 0.777 & Non-Fickian transport \\
\hline F6 & 0.968 & 0.940 & 0.929 & 0.777 & Non-Fickian transport \\
\hline F7 & 0.967 & 0.899 & 0.906 & 0.729 & Non-Fickian transport \\
\hline F8 & 0.934 & 0.934 & 0.955 & 0.638 & Non-Fickian transport \\
\hline F9 & 0.909 & 0.773 & 0.936 & 0.605 & Non-Fickian transport \\
\hline F10 & 0.892 & 0.873 & 0.940 & 0.572 & Non-Fickian transport \\
\hline F11 & 0.968 & 0.940 & 0.929 & 0.777 & Non-Fickian transport \\
\hline F12 & 0.839 & 0.970 & 0.990 & 0.538 & Non-Fickian transport \\
\hline $\mathrm{F} 13$ & 0.898 & 0.917 & 0.956 & 0.566 & Non-Fickian transport \\
\hline $\mathrm{F} 14$ & 0.968 & 0.940 & 0.929 & 0.777 & Non-Fickian transport \\
\hline F15 & 0.889 & 0.957 & 0.975 & 0.552 & Non-Fickian transport \\
\hline F16 & 0.851 & 0.943 & 0.971 & 0.532 & Non-Fickian transport \\
\hline F17 & 0.947 & 0.943 & 0.934 & 0.690 & Non-Fickian transport \\
\hline
\end{tabular}

\subsection{Formulation development}

In the development of any pharmaceutical formulation, a very important issue is to design a formulation with the optimized quality. The RSM has been ordinarily used for the designing and optimization of the various pharmaceutical formulations, which needs minimum experimentation. Thus, it is less time consuming and cost effective than the other typical ways of formulating the dosage forms. Based on the design of experiments, RSM encompasses the generation of polynomial equations of the response over the experimental domain to determine the optimum formulation(s). A computer aided optimization technique was employed to investigate the formulation design by using Box-behnken Design Expert Software version 8.0.7.1. and studies the effect of concentration of various polymer blends used on the properties like floating lag time (FLT), cumulative percent drug release (\%CDR) of CPL gastroretentive floating tablets.

\subsection{Optimization}

The FLT and \%CDR data (Table 17) was entered into the generated design model and then the software generates model graphs to interpret and evaluate the given data to find out the best response. Response 1 (FLT in $0.1 \mathrm{~N}$ ( $\mathrm{pH} 1.2$ ) $\mathrm{HCl})$ and $2(\% \mathrm{CDR}$ in $0.1 \mathrm{~N}(\mathrm{pH} 1.2) \mathrm{HCl})$ were analyzed by ANOVA for Response Surface Linear Model-1 and 2 respectively.

\subsubsection{Response 1: FLT in 0.1 N HCl (pH 1.2)}

The Model F-value of 39.24 implies the model is significant. There is only a $0.01 \%$ chance that a "Model F-Value" could occur in large due to noise. 


\subsubsection{Response 2: \%CDR in 0.1 $\mathrm{N} \mathrm{HCl}(\mathrm{pH} \mathrm{1.2)}$}

The Model F-value of 40.51 implies the model is significant. There is only a $0.01 \%$ chance that a "Model F-Value" could occur in large due to noise. All responses were fitted to linear models as suggested by Design expert software 8.0.7.1. The $\mathrm{F}$ value for FLT, \%CDR were found to be $39.24,40.51$ respectively indicating that the models are significant. The values of Prob $>$ F were found to be $<0.0001$ for all responses indicating that the models are significant (Table 7). The contour and response surface plots for all responses of all formulation factors are shown in figure 2. With the help of these $3 \mathrm{D}$ and contour graphs, the point at which maximum predicted response shown was recorded. With the help of factors tool, the concentrations of critical factors were adjusted in the software to show the maximum predicted response (Table 8).
Table 7. ANOVA for Response Surface Linear Model-1 \& 2

\begin{tabular}{lcccccc}
\multicolumn{1}{c}{ Source } & df & \multicolumn{2}{c}{ Model-1 } & \multicolumn{2}{c}{ Model-2 } & Significance \\
& & F Value & $\begin{array}{c}\text { Prob }> \\
\text { F }^{* *}\end{array}$ & F Value & $\begin{array}{c}\text { Prob }> \\
\text { F }^{* *}\end{array}$ & \\
\hline Model & 9 & 39.24 & $<0.0001$ & 40.51 & $<0.0001$ & Significant \\
HPMC K4M & 1 & 297.30 & $<0.0001$ & 291.46 & $<0.0001$ & Significant \\
CA & 1 & 16.91 & 0.0045 & 17.34 & 0.0042 & Significant \\
SBC & 1 & 14.28 & 0.0069 & 10.03 & 0.0158 & \\
Lack of Fit & 3 & & & & & Valid \\
Pure Error & 4 & & & & & Valid \\
\hline
\end{tabular}

${ }^{*}$ A recommendation is a minimum of 3 lack of fit $\mathrm{df}$ and $4 \mathrm{df}$ for pure error.This ensures a valid lack of fit test.

**Values of "Prob > F" less than 0.0500 indicate model terms are significant and Values greater than 0.1000 indicate the model terms are not significant.
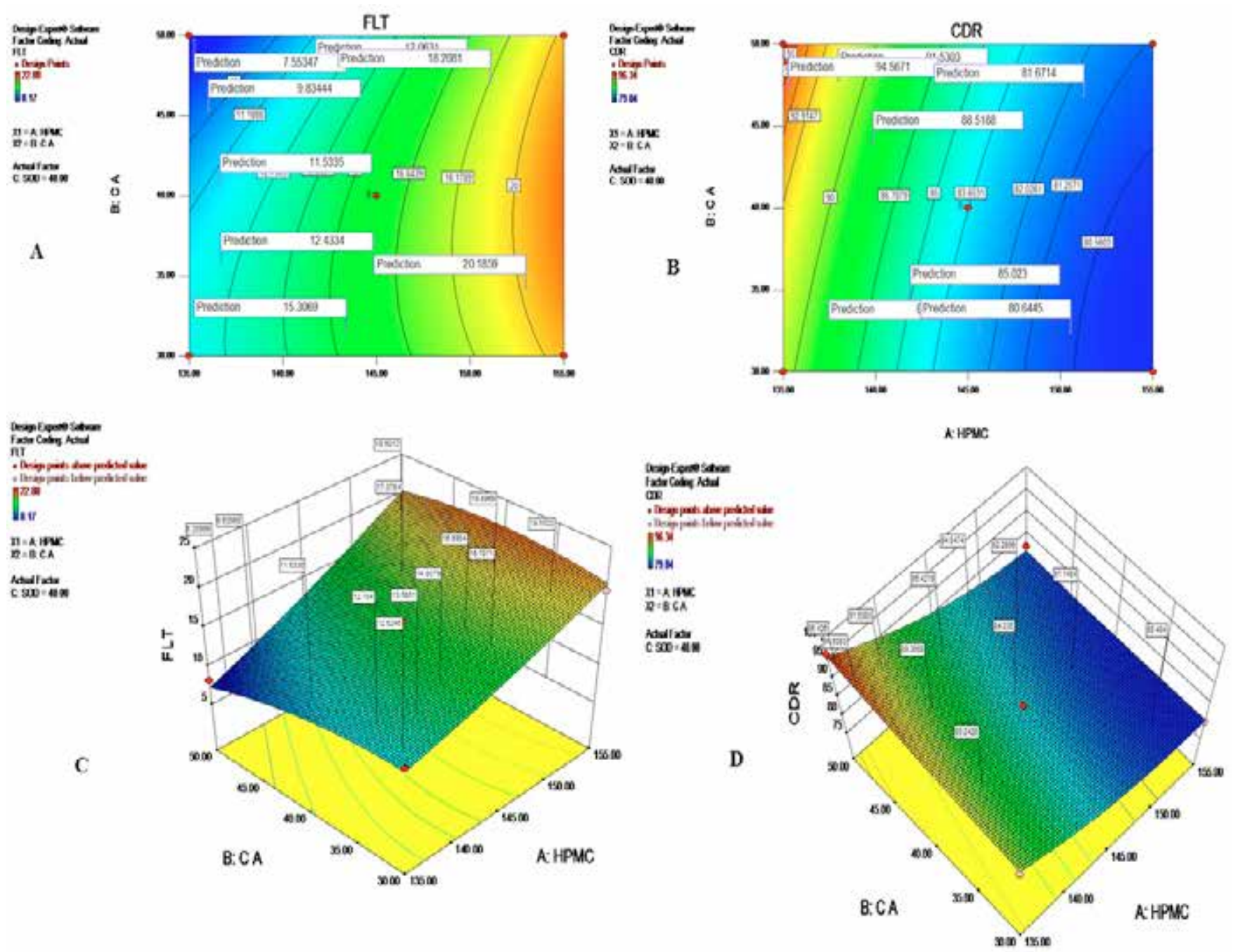

Figure 2. Correlation between experimental and predicted values. A) Contour plot of FLT in $0.1 \mathrm{HCl}(\mathrm{pH}$ 1.2) buffer, B) Contour plot of \% CDR, C) 3D Graph for FLT in $0.1 \mathrm{HCl}$ (pH 1.2) buffer, D) 3D Graph for \% CDR. 


\subsubsection{Point Prediction and optimization results}

To optimize all the responses with different targets, a multicriteria decision approach (a numerical optimization technique by the desirability function and a graphical optimization technique by the overlay plot) was used. The optimized formulation was obtained by applying constrains on dependent variable responses and independent variables. Constrains were FLT in $0.1 \mathrm{~N}(\mathrm{pH} 1.2) \mathrm{HCl} ; \% \mathrm{CDR}$ at $8 \mathrm{~h}$ and these constrains are common for all the formulations.
The recommended concentrations of the independent variables were calculated by the Design expert software from the above plots which has the highest desirability near to 1.0. The predicted concentrations of critical factors (HPMC K4M-136.08 mg, CA-30.11 mg, SBC-49.57 mg) generated by software showed the FLT of $7.856 \mathrm{sec}$ and drug release of 99.15\% (Table 8). Based on these predictions, an optimized formulation has been prepared with the predicted variable factors and analyzed for the \%CDR and FLT.

Table 8. Maximum predicted response (Two-sided Confidence $=95 \%(n=1)$ ) and Percentage Prediction Error of the Optimized formulation

\begin{tabular}{cccccccc}
\hline Response & Prediction & SD & SE Predicted & 95\% PI low & 95\% PI high & $\begin{array}{c}\text { Experimental } \\
\text { Value }\end{array}$ & $\begin{array}{c}\text { Percentage Prediction } \\
\text { Error }\end{array}$ \\
\hline FLT & $7.856 \mathrm{Sec}$ & 0.8786 & 1.350 & 1.9741 & 8.36027 & $8.041 \mathrm{sec}$ & -2.166 \\
$\% \mathrm{CDR}$ & $99.151 \%$ & 1.0350 & 1.5907 & 93.3951 & 101.458 & $97.691 \%$ & -1.495 \\
\hline
\end{tabular}

\subsubsection{Evaluation and validation of the optimized formulation}

By taking the predicted factors into consideration, a formulation has been developed. The prepared formulation was evaluated for the precompressional and post compressional parameters. Precompression parameters like angle of repose, Hausner's ratio and Carr's index were found to be within the prescribed limits. Post compressionl parameters like the weight variation, thickness, drug content, swelling index, FLT, In-vitro dissolution studies were also studied. The tablets produced with the predicted concentrations of critical factors showed drug release of $97.691 \%$ in $8 \mathrm{~h}$ and FLT of $8.041 \mathrm{sec}$ (Table 9) and the results are in very close agreement with the model predictions. Drug release from the optimized formulation F18 followed zero order release $\left(\mathrm{r}^{2}=0.916\right)$ with Non-fickian type of diffusion mechanism $(n=0.505)$. The relative error (\%) between the predicted and experimental values confirms the predictability and validity of the model.

Table 9. Evaluation of optimized formulation (F18)

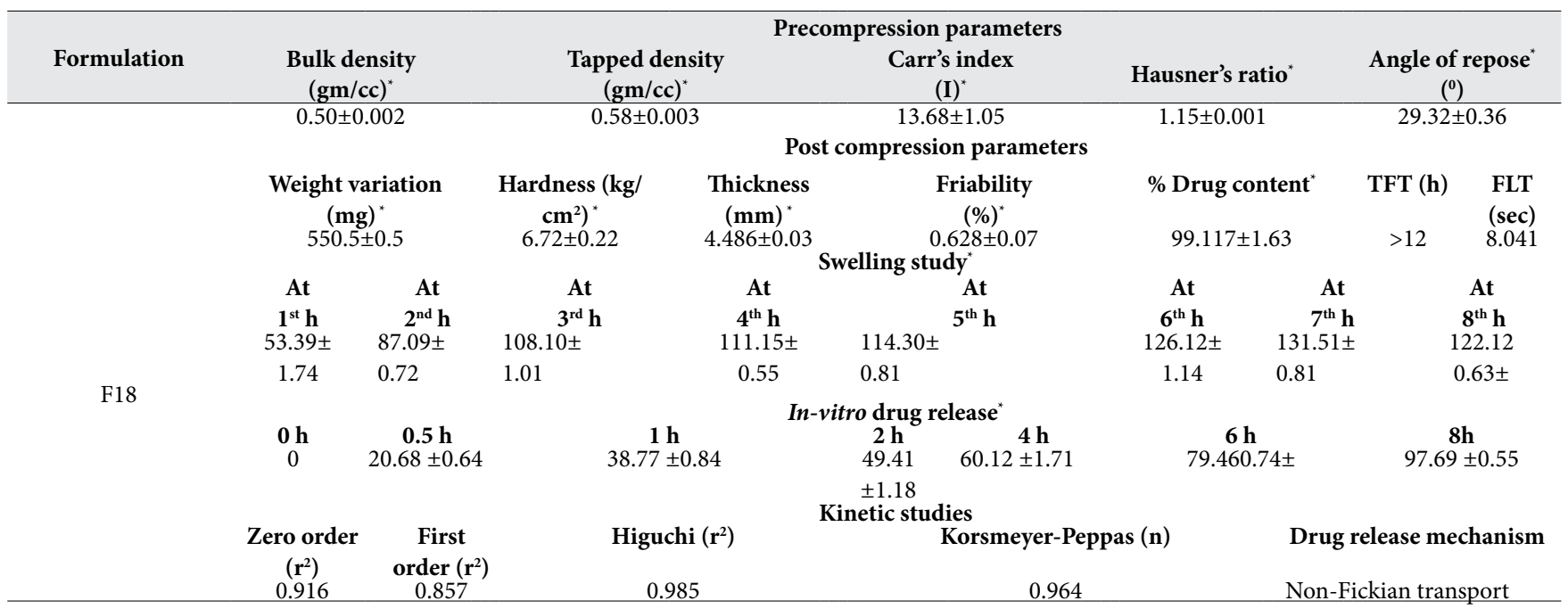

${ }^{*}$ All the results were expressed in mean \pm SD $(n=3)$

\section{CONCLUSION}

From the experimental data, it could be concluded that a successful gastro retentive floating drug delivery system for CPL has been developed by direct compression method using Box-behnken RSM. Statistically optimized formulation containing CPL showed promising results and there exist a scope for in-vivo evaluation using suitable animal models and increase in bioavailability may be confirmed.

\section{CONFLICT OF INTEREST}

The authors report no conflict of interests. The author along are responsible for content and writing of paper. 
Sefaleksin içeren midede kalış süresini uzatan yüzen tabletlerde uzatılmış salınım özelliklerinin geliştirilmesi ve istatistiksel değerlendirilmesi

\section{ÖZ}

Bu çalışmada, Sefaleksin (CPL) içeren, uzatılmış salınım özelliğine sahip, midede kalış süresini uzatan yüzen tabletlerin direkt basım yöntemi ile formülasyonu ve Box-behnken Uyarıcı Yüzey Yöntemine (RSM) göre optimizasyonu amaçlanmıştır. Bilgisayar ortamında Box-behnken RSM (Design Expert Sotware version 8.0.7.1.) yöntemi kullanılarak; çeşitli polimer karışımlarının derişimlerinin, CPL içeren midede kalış süresini uzatan yüzen tabletlerin yüzme gecikme zamanı (FLT) ve kümülatif ilaç salınım yüzdesi (\%CDR) üzerine etkileri çalışılmıştır. Üzerinde çalışılan bağımsız değişken; polimer derişimleri (X1), bağımlı değişkenler ise $0,1 \mathrm{~N}$ $\mathrm{HCl}(\mathrm{pH} 1,2)$ tamponu içerisinde FLT (Y1) ve \%CDR (Y2) olarak belirlenmiştir. Basım öncesi çalışmalarda, hazırlanan 17 formülasyonun ve optimize edilen formülasyon olan F18'in farmakopede belirtilen gerekliliklerle uyumlu olduğu belirlenirken basım sonrası parametrelerin de kabul edilebilir olduğu tespit edilmiştir. Bilgisayar ortamında tahmin edilen FLT ve \%CDR verileri, optimize edilen formülasyon olan F18'in deneysel yöntemlerle elde edilen FLT ve \%CDR sonuçları ile karşılaştırılmıştır. F18'in deneysel sonuçlarının bilgisayar ortamında yapılan tahminler ile yüksek oranda benzerlik gösterdiği ve diğer 17 formülasyonla karşılaştırıldığında F18'in daha iyi FLT ve \%CDR değerlerine sahip olduğu görülmüştür.

Anahtar kelimeler: Sefaleksin; Design Expert Yazılımı; Yüzen tabletler; Optimizasyon; Uyarıcı Yüzey Yöntemi.

\section{REFERENCES}

1. Amit KN, Ruma M, Biswarup D. Gastro retentive drug delivery systems: a review. Asian J Pharm Clin Res 2010; 3: 2-10.

2. Sunil K, Faraz Jl, Meenu R, Saurabh S. Gastro Retentive Drug Delivery System: Features and Facts. Int J Res Pharm Biomed Sci 2012;3:125-36.

3. Ravi Kumar Z, Snehalatha, Nagaraja TS, Yogananda R. Formulation, Evaluation and study of effect of hydrophilic polymers on release rate of Cephalexin floating tablets. Int J Drug Discov Herbal Res 2013;3:541-8.

4. Jishnu V, Prabhakaran R1, Gilhotra RM. Formulation and evaluation of Cephalexin extended release matrix tablets using $3^{2}$ factorial design. J Young Pharmacists 2011;3: 259-66.

5. Radha Krishna N, Deepika B, Chinna Eswaraiah M. Formulation and in-vitro evaluation of floating matrix tablets of Cephalexin. Int J Pharm Chem Sci 2013;2:1519-27.

6. Mohammed AH, Yeswanth Reddy M, Maimuna A. Formulation and evaluation of gastro retentive effervescent floating matrix tablets of Cephalexin. J Drug Deliv Therap 2014;4:22-30.

7. Sreenivasa Rao K, Rakesh RV, Dattatreya BU, Praveen SP, Karankumar VB. Development and evaluation of gastroretentive floating tablets of Cefpodoxime Proxetil. Int J Res Pharm Chem 2012;2: 46-53.

8. Kamini V, Rajesh KS, Lalit LJ. Formulation and evaluation of floating microspheres of Cephalexin. Int J Pharm Sci Rev Res 2011;11: 69-75.

9. Mohammed AH, Yeswanth Reddy M, Maimuna A. Formulation and evaluation of gastro retentive effervescent floating matrix tablets of Cephalexin. J Drug Del Ther 2014;4: 22-30.

10. Shah D, Shah Y, Rampradhan M. Development and evaluation of controlled release diltiazem hydrochloride microparticles using cross-linked poly (vinyl alcohol). Drug Dev Ind Pharm 1997;23:567-74.

11. Venkateswarlu K, Shanthi A. Formulation and evaluation of glipizide matrix. IOSR J Pharm Biol Sci 2012;2:17-23.
12. Khan KA. The concept of dissolution efficiency. J Pharm Pharmacol 1975;27:48-9.

13. Dave BS, Amin AF, Patel MM. Gastroretentive drug delivery system of ranitidine hydrochloride: Formulation and In-Vitro Evaluation. AAPS PharmSciTech 2004;5:77-82.

14. Roy P, Shahiwala A. Statistical optimization of Ranitidine Hydrochloride floating pulsatile delivery system for chronotherapy of nocturnal acid breakthrough. Eur J Pharm Sci 2009;37:363-9.

15. Rajeshwar KKA, Singh R, Vijay J. Mucoadhesive microspheres of famotidine: preparation characterization and in vitro evaluation. Int J Eng Sci Tech 2010;2:1575-80.

16. Anil kumar JS, Arun NW, Manoj BP, Harinath NM. Formulation and In vitro evaluation of sustained release floating tablet of Cephalexin using hydrophilic polymers. Int J Pharm Sci 2010; 2: 58-65.

17. Urmi D, Mohammad SH. Effects of release modifier on Carvedilol release from kollidon SR based matrix, Int Curr Pharm J 2012;1:186-92.

18. Suvakanta D, Narasimhamurthy P, Lilakanthnath, Chaudhary P. Kinetic modeling on drug release from controlled drug delivery systems. Acta Pol Pharm 2010;67:217-33.

19. Prajapati S.T, Patel LD and Patel DM. Gastric floating matrix tablets: design and optimization using combination of polymers. Acta Pharm 2008;58:221-9.

20. Ulrike B, Roland B. In situ gelling, bioadhesive nasal inserts for extended drug delivery: In vitro characterization of a new nasal dosage form. Eur J Pharm Sci 2006;27:62-71.

21. Viridena A, Wittgrenb B, Larsson A, Investigation of critical polymer properties for polymer release and swelling of HPMC matrix tablets. Eur J Pharm Sci 2009;36:297-309. 